\title{
The current problem of traffic congestion on highway
}

\author{
Yanqin Huang \\ School of North China Electric Power University, Baoding 071000, China \\ 1041087586@qq.com
}

Keywords: Toll plaza, Throughput, The Queuing theory, Poisson distribution.

\begin{abstract}
In this paper, we try to analyses the current problem of traffic congestion in highway. Taking highways in New Jersey of the United States for example, the Queuing theory and Poisson distribution are used to assess the performance of the current toll plaza. The queuing theory is used to calculate the throughput. And the average number of waiting cars can be obtained based on the theory of Poisson distribution
\end{abstract}

\section{Introduction}

At most time, the highway in New Jersey has a large traffic, but the utilization of toll plaza badly obstructs the travel speed ${ }^{[1]}$. In order to determine the proportional weights of the corresponding factors in distributing the different types of tollbooths ${ }^{[4]}$. We try to do some studies on the research of the highway toll plaza.

\section{Assessment of the current toll plaza}

In order to assess the toll plaza's ability of accident prevention and throughput, we firstly consider that the proportion of human-staffed tollbooth and electronic tollbooth is 3:5. Considering the different types of vehicle, we identify that the proportion of car and truck is 39:11. In this way, the throughput and the average number of waiting vehicles can be calculated.

\section{Analysis the throughput of the toll plaza through queuing theory}

Before calculating the throughput, we need to know the traffic situation. In this way, the DHV should be calculated first.

DHV, also called traffic volume, it can be calculated from that

$D H V=A A D T \times K \times D$

Where AADT means annual average daily traffic, $\mathrm{K}$ means the ratio of the 30 th hour traffic volume and AADT, under normal circumstances, it take the value of 0.12 . D means the unbalanced distribution coefficient of the traffic volume direction, and its standard value is 0.6. Usually, AADA is calculated according to the projected value. According to the statistics of the related authority, the value of AADA is determined as $28900 \mathrm{pcu} / \mathrm{d}$.

After the DHV is calculated, the average time of the following coming vehicle a can be solved from that

$$
a=\frac{3600}{D H V}
$$

Where $\lambda$ means the service time when passing the tollbooth, according to the empirical statistic, its average value $\bar{\lambda}$ can be determined as 14 seconds.

Because of the large weight, longer length and weight charges of large vehicles, resulting in its service time longer than small cars. Usually, take small cars as the standard to calculate the maximum throughput of toll plaza. In this way, the number of the tollbooth can be designed. So we need to forecast the toll plaza in the large car service time relative to the conversion coefficient of small cars converted. The traffic volume after converted is viewed as the standard. 
The time frequency distribution of different type of vehicle is different. In the field, related survey found that the conversion coefficient of service time for large cars is relative to small cars. Thus, we assume that the conversion coefficients are the same for different type of vehicles.

View the occupancy of different types of vehicles as Ri, we can obtain the table below:

Table 1. Converted service hours

\begin{tabular}{|c|c|c|}
\hline Type of vehicle & Occupancy $R_{i}$ & conversion coefficient $A_{i}$ \\
\hline Large trucks & $R_{1}$ & 1.5 \\
\hline Small cars & $R_{2}$ & 1 \\
\hline Small cars using E- ZPass & $R_{3}$ & 0.8 \\
\hline $\begin{array}{l}\text { Small cars using } \\
\text { Exact-change }\end{array}$ & $R_{4}$ & 0.9 \\
\hline
\end{tabular}

In this way, the service time can be calculated from that

$\lambda=\sum R_{i} A_{i} \bar{\lambda}$

Where $\lambda$ means the service time when passing the tollbooth, $\bar{\lambda}$ means the average value of $\lambda$. According to the empirical statistic, its average value can be determined as 14 seconds.

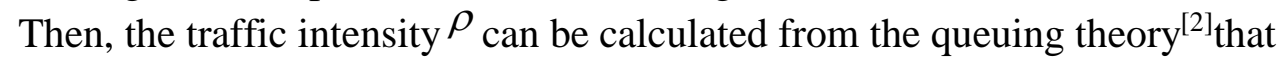

$\rho=\frac{\lambda}{a}$

The traffic intensity of the single tollbooth is $\mu$, and it can be got from that

$\mu=\frac{\rho}{B}$

After calculating the traffic intensity of the single tollbooth ${ }^{\mu}$, we assume that the average number

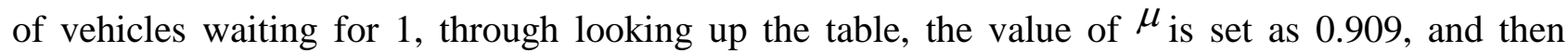
compared with the actual value and found that the actual maximum throughput $\mathrm{T}$ far exceeds the set maximum rated throughput. The service time $\lambda$ and the number of tollbooth $\mathrm{B}$, the throughput of the toll plaza can be determined from that

$$
T=\frac{3600 \mu}{\lambda} B
$$

Substitute the specific values, the value of traffic intensity of the single tollbooth $\mu_{\text {is }} 0.9882$, and the value of throughput can be finally calculated that 2081 vehicles will come to the toll plaza per hour. However, the rated maximum throughput is 1914, so it cannot satisfy the large traffic volume.

\section{Calculate the average number of waiting cars based on Poisson distribution}

Queuing model is utilized to calculate the average number of waiting cars. The whole process of toll is composed of vehicle arriving rate, queuing system and service rate. The vehicle arriving rate is random. Most researches suggest that the arrival distribution of vehicles is subject to Poisson distribution $^{[4]}$. So the process of vehicle arrival is a Poisson process. And the traffic intensity is subject to Poisson distribution, too. Thus, in a queuing system, the probability of the situation where there is no car passing the toll plaza is that

$$
P(0)=\frac{1}{\left[\sum_{n=0}^{k-1} \frac{\rho^{n}}{n !}+\frac{\rho^{B}}{B(1-\mu)}\right]}
$$

So the average waiting time $\mathrm{w}$ in queuing is that

$$
w=\frac{\rho^{B}}{B ! B} \frac{\lambda}{(1-\mu)^{2}} P(0)
$$

Where $\lambda$ means the service time when passing the tollbooth, its value is determined as 13.678 seconds. Finally, the average number of the waiting cars for a single tollbooth q can be got from that 


$$
q=\frac{w}{b}
$$

Substitute the specific values, the value of the average number of the waiting cars for a single tollbooth $\mathrm{q}$ can be got that $\mathrm{q}=40.6$. Far more than the $\mathrm{q}=1$ setting.

\section{Gray Correlation Analysis for the correlation degree of each factor}

From the analyse of the current toll plaza we can see that the barrier toll has badly affected the highway capacity. In order to come up with an improved design, we need to analyze the related factors and calculate the correlation degree using gray correlation analysis.

When constructing a toll plaza, the factors to consider are that throughput, accident rate, service time, the average waiting time for vehicles and cost. As for a completed toll plaza, the cost is no use to be considered, thus, we just analyze the remaining factors.

In the official website, we obtain the relative statistics for the corresponding factors of the toll plaza $^{[5]}$. In order to reflect the system behavior characteristics, the reference sequence is introduced. It can be expressed from that

$$
x_{0}=\left\{x_{0}(k) \mid k=1,2, \cdots, n\right\}
$$

In order to compare the how the different factors effect the system, the comparison sequence is introduced. And it can be expressed from that

$$
x_{i}=\left\{x_{i}(k) \mid k=1,2, \cdots, n\right\}, i=1,2, \cdots, m
$$

Because the data in each factor column of the system may be different in dimension, so it is difficult to compare or to obtain the correct conclusion. As a result, it is supposed to carry out the non-dimensional data processing when using gray correlation analysis. Through dividing with the reference value, the non-dimensional data processing can be carried out from that

$$
x_{i}(k)=\frac{X_{i}(k)}{X_{i}(k)},(k=1,2, \cdots, n ; i=0,1,2, \cdots, m)
$$

Correlation coefficient can represent the strength of the association between two variables. It can be calculated from that

$$
\xi_{i}(k)=\frac{\min _{i} \min _{k}\left|y(k)-x_{i}(k)\right|+\rho \max _{i} \max _{k}\left|y(k)-x_{i}(k)\right|}{\left|y(k)-x_{i}(k)\right|+\rho \max _{i} \max _{k}\left|y(k)-x_{i}(k)\right|}
$$

Where $\rho$ means resolution factor, it satisfies that $\rho>0$. When $\rho$ gets smaller, the resolution will get greater. In general, the interval of $\rho$ is $(0,1)$. To obtain the best resolution, the value of $\rho$ is usually taken as 0.5 .

The correlation coefficient is the value of the correlation degree between the comparison sequence and the reference sequence at each moment. When it is determined, the relevance ri between the two sequences can be got from that

$$
r_{i}=\frac{1}{n} \sum_{k=1}^{n} \xi_{i}(k), \quad k=1,2, \cdots, n
$$

After the correlation coefficient is calculated, we can compare similarity between the comparison sequence and the reference sequence through sorting by the value of correlation coefficient. Take an example, if $\mathrm{r} 1>\mathrm{r} 2$, the conclusion is that the reference sequence is more similar to the comparison sequence $\mathrm{x} 2$.

\section{Conclusion}

Through MATLAB software, we can see that the traffic volume and the number of tollbooth have the bigger correlation coefficient with the site rating, and the number of waiting cars and accident times have the smaller correlation coefficient. Both the traffic volume and the number of tollbooth can effectively affect the throughput. Furthermore, considering the outcome of the assessment, we 
have known that the barrier toll has badly affected the throughput. In this way, if we want to improve the site rating of a toll plaza, we need to put the throughput in the first place.

\section{References}

[1]. ANALYSIS OF THE TEN YEAR PLAN TO REMOVE TOLL BARRIERS FROM THE NEW JERSEY GARDEN STATE PARKWAY August 1st, 2002.

[2]. Su Li, "Study on the design of highway toll station", A Dissertation Submitted for the Degree of Master, U41;U2 10710-2009121334.

[3]. Mohammad Motie and Ketan Savla, "Throughput Analysis of a Horizontal Traffic Queue under Safe Car Following Models", 2016 IEEE 55th Conference on Decision and Control (CDC), ARIA Resort \& Casino, December 12-14, 2016, Las Vegas, USA.

[4]. Wu Xia, Limin Cai, Zhongyuan Lai, Shizhen Zhang and Zhijun Yao, "Hazardous Materials Transportation Routing: Using Fuzzy Analytic Hierarchy Process Based on Historical Traffic Data", The 2016 3rd International Conference on Systems and Informatics (ICSAI 2016).

[5]. https://foursquare.com/v/bergen-toll-plaza/4b9eb66ff964a520b8fb36e3 